\title{
Novel technique using Surgical Scrub Sponges to protect the nose and face during prone ventilation
}

\author{
Thomas Stubington ${ }^{1}$ and Mohammed Mansuri ${ }^{1}$ \\ ${ }^{1}$ Royal Derby Hospital
}

May 6, 2020

\section{Introduction}

COVID 19 in particular affects the lungs causing an ARDS type picture resulting in an atypical form of ARDS whereby there is disproportionately poor oxygenation despite reasonably preserved lung compliance in the early stages ${ }^{1}$. Experience from Italy and China suggests that nursing the patient in a prone position is potentially beneficial and can improve outcomes when carried out in the early stages of the disease ${ }^{1,2}$. This has resulted in its inclusion in several international guidelines and adoption around the world as a valid intervention for COVID 19 patients $^{3}$

Proning is not a new phenomenon and has been used as a treatment option for ARDS for over 20 years. It is not without complications and as well as the displacement of tubes and lines, the exacerbation of existing traumas or dehiscence of surgical wounds there are also reports of pressure necrosis secondary to prone positioning particularly of the face and nose ${ }^{4} \mathrm{~A}$ cochrane review in 2015 concluded that prone ventilation was directly responsible for an increased risk of pressure sores ${ }^{5}$ There is some suggestion that the pressure damage caused by proning occurs regardless of preventative measures put in place (such as foam supports and measures to relieve pressure $)^{4}$ But it also seems that this pressure damage is often mild and self resolving ${ }^{6}$. Regular repositioning of the head may also reduce pressure damage accordingly ${ }^{7}$

Given that larger numbers of patients are likely to be proned and that proning is directly linked to pressure damage to the face and nose it would seem logical that this would represent an increase in referrals to ENT to assess this. Anecdotally this is the case in our department where we have received several such calls having never previously encountered this complication in routine practice. Although patients should be proned with the head turned to one side to avoid such pressure damage ${ }^{8}$ due to the highly unstable nature of COVID 19 patients and in some cases limited cervical spine rotation inevitably some patients will end up in positions where there nose is at risk. We present our approach to the management of these injuries borrowing from theory and practice used to manage patients having undergone rhinological procedures.

\section{Technique}

Our approach focuses on the following:

1. Easily accessed readily available materials - Given the demand healthcare services are under around the world a complex and expensive solution requiring multiple bespoke components is not desirable.

2. Respectful of surrounding structures - If not applied with consideration of surrounding structures you risk swapping nasal injury for corneal or labial 
3. Easily Monitored - Proning presents a significant challenge for visualisation of the nose and eyes, bulky padding risks obscuring the affected area preventing monitoring. Skin breakdown and infection are a real concern with pressure damage in this area.

4. Easily Adapted - A bespoke solution for each patient may be effective but will also be expensive and likely to take time to produce/acquire

Surgical sponge has already been described as an appropriate material to immobilise and protect delicate grafts ${ }^{9}$ and it is our practice to also make use of it in the early stages of recovery post rhinectomy. It is our opinion that surgical scrub sponges meet the criteria set out above and represent an effective method of pressure relief in patients being ventilated prone:

1. Easily accessed readily available material - these sponges are available in all operating theatres and are bought in bulk at relatively low cost

2. Respectful of surrounding structures - the sponges can be adjusted and cut to avoid nearby structures

3. Easily monitored - the 3 part design allows the sponge to be moved and the nose viewed

4. Easily Adapted - can be cut to any size and additional sponges can be added to account for larger anatomy

The steps taken to measure and apply the sponge are detailed in figure 1.

The solution can be seen in figure 1 and2. Figure 2 demonstrates that because the sponges are only secured together at one edge they can splay slightly allowing pressure to be re-distributed and stopping the sponge bulging and encroaching on the eyes.

\section{Conclusion}

We describe a simple technique to relieve pressure from the nose and face in patients who are ventilated prone. The technique focuses on low cost easily available materials, that allow easy monitoring of the area and is easily adapted to the individual patient. With the increasing use of prone ventilation to treat COVID19 it is our feeling that this may reduce the incidence of proning induced pressure injury to the face and nose.

\section{Key Points}

- COVID-19 has resulted in increasing numbers of patients ventilated prone

- A known complication of proning is pressure damage to the nose and face

- Although patients should ideally be positioned with the head turned this is not always possible

- Techniques to relieve pressure from the face and nose are essential and can be achieved with a simple and low cost intervention

\section{Figure Legends}

Figure 1: Step by step approach to cut surgical sponge to appropriate size and fit to the patients face

Figure 2: Lateral view of the sponge in situ

Figure 3: Top down view of the sponge in situ this demonstrates the slight splaying of the three sponges to distribute pressure. 


\section{Bibliography}

1 Gattinoni L., Coppola S., Cressoni M., et al. (2020) Covid-19 Does Not Lead to a "Typical" Acute Respiratory Distress Syndrome.

Am. J. Respir. Crit. Care Med.

2 Meng L., Qiu H., Wan L., et al. (2020) Intubation and Ventilation amid the COVID-19 Outbreak: Wuhan's Experience.Anesthesiology .

3 Alhazzani W., Møller M.H., Arabi Y.M., et al. (2020) Surviving Sepsis Campaign: guidelines on the management of critically ill adults with Coronavirus Disease 2019 (COVID-19). Intensive Care Med.

4 Offner P.J., Haenel J.B., Moore E.E., et al. (2000) Complications of prone ventilation in patients with multisystem trauma with fulminant acute respiratory distress syndrome. In Journal of Trauma - Injury, Infection and Critical Care pp. 224-228. Lippincott Williams and Wilkins.

5 Bloomfield R., Noble D.W. \& Sudlow A. (2015) Prone position for acute respiratory failure in adults. Cochrane Database Syst. Rev. 2015 .

6 Mancebo J., Fernández R., Blanch L., et al. (2006) A multicenter trial of prolonged prone ventilation in severe acute respiratory distress syndrome. Am. J. Respir. Crit. Care Med. 173 , 1233-1239.

7 Messerole E., Peine P., Wittkopp S., et al. (2002) The pragmatics of prone positioning. Am. J. Respir. Crit. Care Med. 165 , 1359-1363.

8 Bamford P., Denmade C., Newmarch C., et al. (2019) Prone Position Guidance in Adult Critical Care .

9 Yiacoumettis A.M., Papadimitriou A.M., Topkas A.T., et al.(2007) Letter: Polyurethane sponge from plastic scrubbing brush used as a pressure dressing over skin graft [5]. Dermatologic Surg. 33 , 1406-1407. 


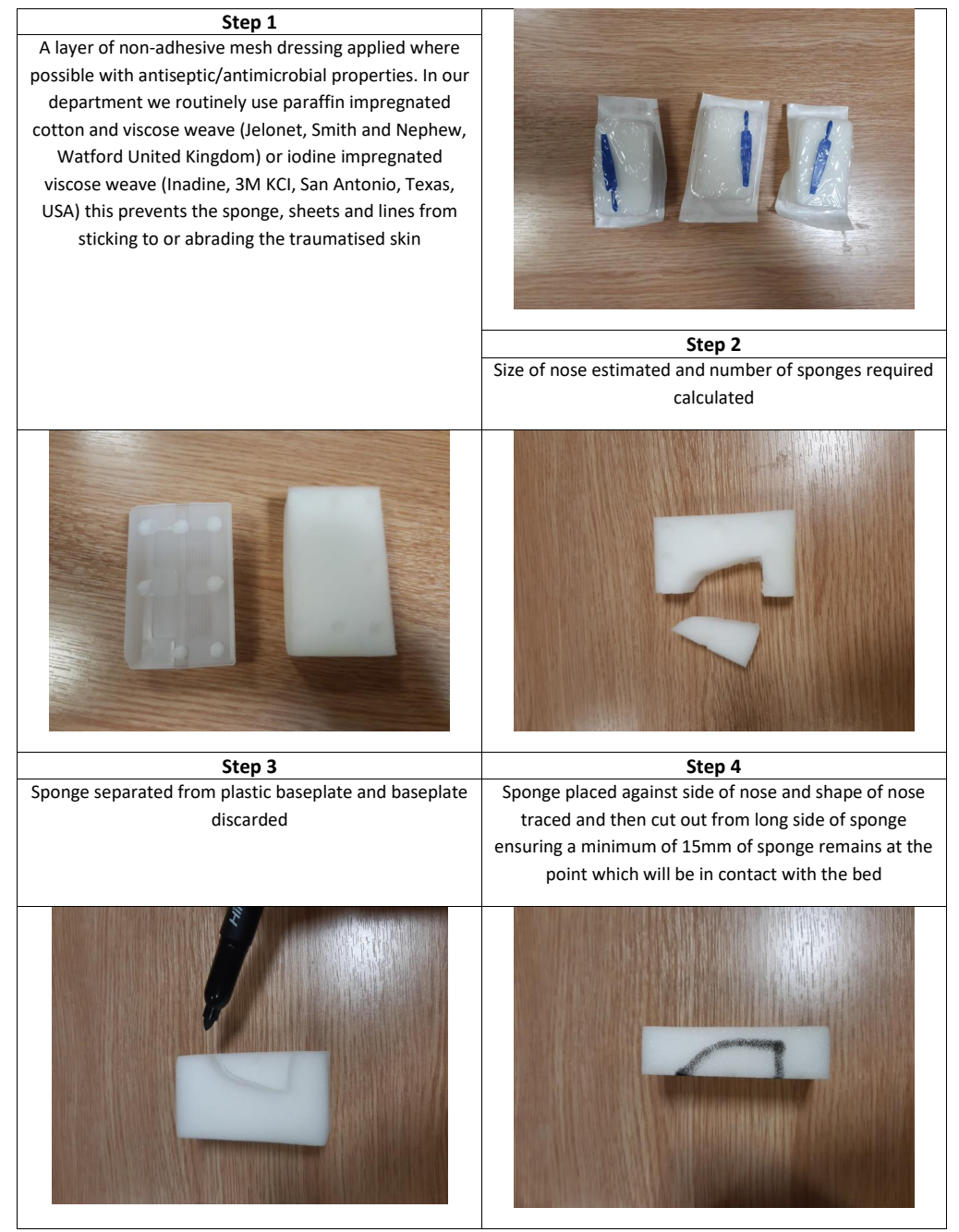



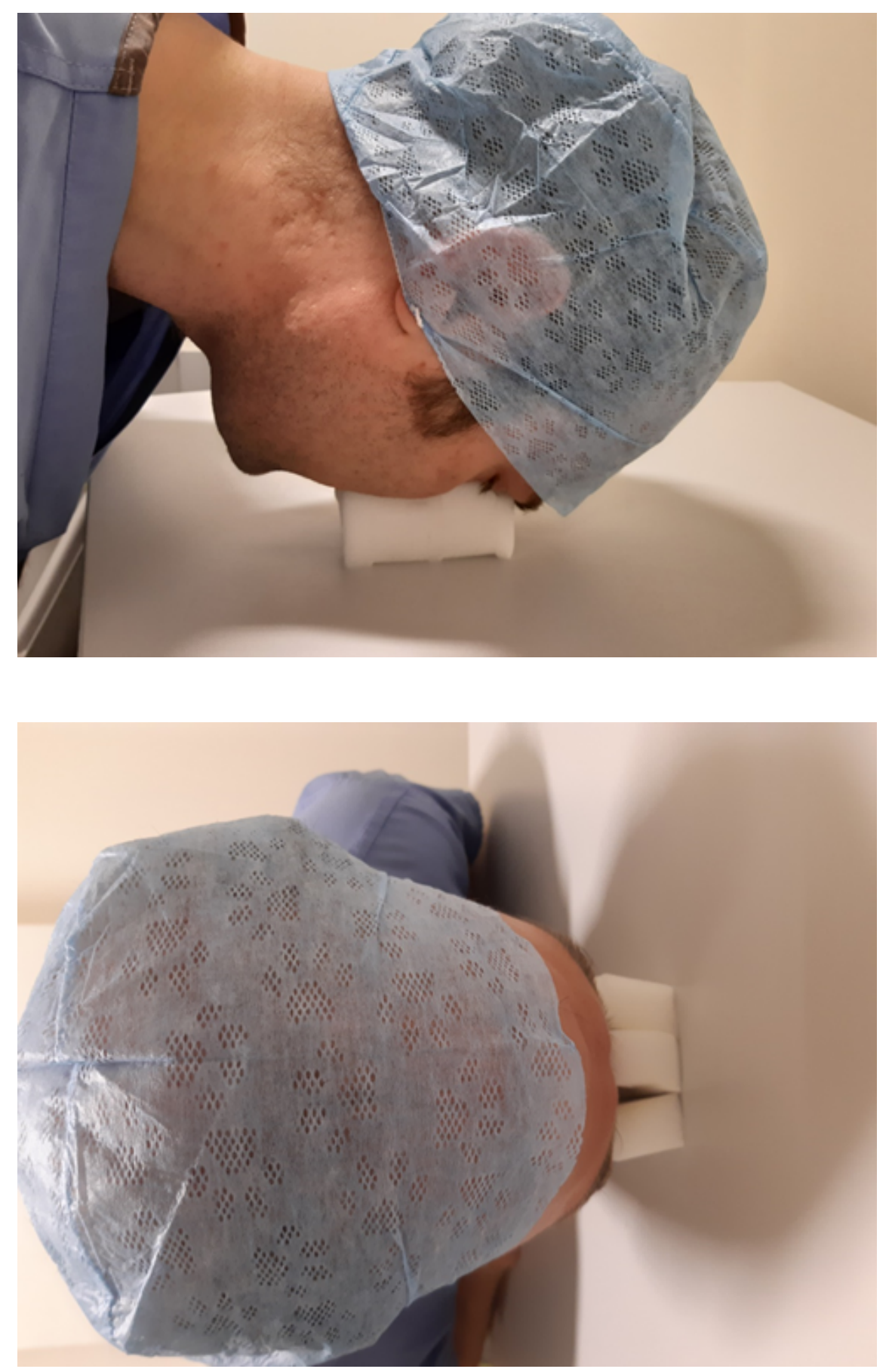